\title{
Development Sustainable Algorithm Optimal Resource Allocation in Information Logistics Systems
}

\author{
Khaled Batiha, PhD \\ Computer science department \\ Al al-Bayt University, Jordan
}

\author{
Safwan Al Salaimeh \\ Computer Science Department \\ Jadara University, Irbid, Jordan
}

\begin{abstract}
This paper deals with new networks technology of corporation system. The data of processing technology choice is actual task in the problem of development a modern computer networks. There are presentation of functions and parameters as a result of this research.
\end{abstract}

\section{Keywords}

logistics, allocation recourse, mathematical model, computer network, parameters.

\section{INTRODUCTION}

Logistics is a science of planning, monitoring, transportation management and storing of finished products and raw materials starting from the buyer and ending with the consumer not to mention the transfer, saving and process of data. The technological logistics system is: Support for these systems, in terms of information and computerized.

The concepts of logistics system are focused on cooperative logistics concepts like Supply Chain Management, Efficient Consumer Response (ECR) or Quick Response (QR). Ni Wang, Jye-Chyi Lu and Paul Kva declared that these concepts are actually discussed in recent logistics publications, were typical descriptions of temporary logistics systems are characterized by notions of push/pull control logic, postponement/decentralization of stock keeping as alternative or complementary strategic opportunities for the design of logistics networks [1,2].

The process of building logistic information system that can be practically used to solve applied economical problems goes through the following phases:

1. To collect the data needed for the use of logistic information systems.

2. To design a mathematical model suitable for the use of logistic information systems.

3. To design a simulation system, introduce applications and achieve the required results.

4. Users interfaces.

\section{RECOURSE ALLOCATION MODEL}

If the optimality criteria option to choose the total profits of the enterprise $\mathrm{F}(\mathrm{x})$, so that the object function can be written as:

$$
F(x)=\sum_{j-1}^{n} c_{j} x_{j} \rightarrow \max
$$

A function limitations, imposed production and technological process, in simplest case is a system of linear and nonlinear algebraic equality. Reflecting the allocation of resources to fund operating time of equipment or availability of financial opportunities

$$
\sum_{j=1}^{n} a_{i j} x_{j} \leq b_{i} \quad(i=\overline{1, m})
$$

Condition, blend to variables can be job with ratio nonnegativity views:

$$
x_{j} \geq 0, \quad(j=\overline{1, n})
$$

Mathematical model (1) - (3) refers to the type simple models of recourse allocation in class linear programming problems. More generally in model (1) -(3) may be include the next recourse constrains:

Recourse constrains equipments $b_{i}$ in types of time funds runtime i-th group

Restriction on the variables related to the range of products $x \mathrm{j}$ in type of range of each variables $d_{1}^{-} \leq x_{j} \leq d_{j}^{+}$ $(j=\overline{1, n})$.

Limit consumption of materials, labor costs and payroll [3].

\section{RECOURSES ALLOCATION \\ ALGORITHM}

Linear programming problem (1) - (3) will deliver the vector matrix

$$
\max \left(C^{T}, X\right) \quad \text { at } A X=B ; X \geq 0 ;
$$

Where $C$ and $X$ - vectors of dimensions $n$;

$A$ - Matrix dimension $\times n$;

$B$ - Vector of dimension .

Classical simplex method operates definite matrix, called simplex table. Each table $T$ has property

$$
T=D^{-1} A ;
$$

Where $D-$ basic square matrix of order $m$ composed of $m$ linearly independent columns of the matrix .

So, if $A=\left[A_{1}, A_{2}, \ldots, A_{m}\right]$, believing that $A_{j}$ represents a column of the matrix $A$, obtain

$$
D=\left[A_{k 1}, A_{k 2}, \ldots, A_{k m}\right],
$$

Where: $k_{j}$ - columns number .

Columns $A_{j}$, matrix components $D$;

From a basic and which called basic where in $k_{j}$ - column of the matrix $T$ consists of zeros, except for one unit in $j$ 
positions believing that $B^{*}=D^{-1} B$, expressions of (4) and (5) obtain the relation $T X=B^{*}$, from which we can conclude that any solution of the system $T X=B^{*}$ is a solution of $A X=B$ vice versa.

Basic feasible solution of support program is $X$, for which $=B^{*} T X=B^{*}, X \geq 0$ and moreover $X_{k j}=b_{j}, j=1, \ldots, m$, and other basic components $X_{k}$ are zeros. While any given columnT. Correspond to some basic plan, and the components $X_{k}$ corresponding columns $A$, are basic components[4,5].

\section{STABLE ALGORITHM SIMPLEX METHOD}

The idea of the inverse matrix is that of the each iteration the search of the optimal solution recalculated no all matrix condition of the problem, as in the usual simplex method only a portion $A_{x}^{-1}$, significantly less than the dimension. Sign of the optimal method of inverse matrix, as in the usual simplex method, acts condition $\Delta_{j} \geq 0, j=\overline{1, n}$.

However, the amount $\Delta_{j}$ determined from the relation

$$
\Delta_{j}=\Lambda A_{j}-C_{j}, \Lambda=C_{x} A_{x}^{-1}
$$

Where $A_{x}^{-1}$ - matrix inverse to the base at the current iteration;

$C_{x}$ - vector of the coefficient of the objective function, meet the basic variables to the current iterations;

$A_{j^{-}} \quad j$ Column vector of the matrix $; C_{j}$ - coefficient of the objective function $j$ variables $j=\overline{1, n}$.

Computational procedure for solving the problem involves filling of the auxiliary and main tables. In table enter the following data of the linear programming problems, decision concerning of the initial support program $X$, the basic components of the initial support program $-X_{i 0}, i=\overline{1, m}$;

Elements $\left\|a_{i j}\right\|_{j=\overline{1, n}}$ - the expansion coefficients of the vectors $A_{j}$ the basic vectors; components of basic vectors in a matrix $A_{x}$; components of the vector $C-\operatorname{line} C_{j}$; magnitude estimates $\Delta_{j}$, defined by the formula (6). Where the main parts of main column, enter the following data [6].

In column $B_{x}$ - number of basic vectors; in column $e_{0}$-values of the basic variables $e_{i 0} i=\overline{1, m}, e_{0}=A_{x}^{-1} A_{0}, e_{i 0}=X_{i 0}$; in columns $e_{1}, e_{2}, \ldots, e_{m}$ - elements $\left\|e_{i j}\right\|_{j=\overline{1, n}}$ matrix $A_{x}^{-1}$; in the last line - components $\lambda_{j}$ vector $\Lambda$, which are calculated by the formula (6). To additional column of the table if the problem is not solved, written vector $A_{k}$, which will be held in basic for which $\Delta_{k}=\min \Delta_{j}$, components of the vector $A_{k}$, the main table is defined by the formula $A_{k}^{o c h}=A_{x}^{-1} A_{k}^{A u x}$, where $A_{k}^{A u x}$ - vector $A_{k}$, taken from the auxiliary tables. If all $a_{i k} \leq 0$, the problem is not taken because of the objective function unbounded certain additional recourses.

In the presence of positive elements $a_{i k}$ determined by the vector which is derived from the basic $\Theta_{r}=\min \left\{\frac{e_{i 0}}{a_{i k}} / a_{i k}>\right.$ $0\}$. let such a vector is a vector $A_{r}$ then $a_{i r}$ - guide element. Next is filled the second all subsequent main table using recurrence relations: $\Theta_{r}=\left\{\begin{array}{lll}e_{i j}^{1}-\frac{e_{r j}^{1}}{a_{r k}} * a_{i k}^{1}, & \text { at } & i \neq r \\ \frac{e_{r j}^{1}}{e_{r k}^{1}}, & \text { at } & i\end{array}\right.$ $i=\overline{1, m}, \quad j=\overline{1, n}$.

Stability of the algorithm provides a check matrix function limitations resulting from or degeneracy followed correction ill condition or singular matrix restrictions.

\section{THE RESULTS}

This paper deals with new networks technology of corporation system. The data of processing technology choice is actual task in the problem of development a modern computer networks. There are presentation of functions and parameters as a result of this research.

\section{REFERENCES}

[1] Dr. Howard Bandy, Modeling Trading System Performance, Blue Owl Press, 2011.

[2] Yan, Houmin; Yin, George; Zhang, Qing, Stochastic Processes, Optimization, and Control Theory: Applications in Financial Engineering, Queuing Networks, and Manufacturing Systems, Springer, USA, 2006.

[3] Guernsey, GY, Process Dynamics: Modeling, Analysis, and Simulation, Prentice Hall, United Kingdom, 2003.

[4] Otto Bretscher, Linear Algebra with Applications, Prentice Hall, UK, 2008.

[5] Silvia T. Akuna, Software processing modeling, Springer, USA, 2005.

[6] Hossein Bidgoli, Modern Information Systems for Managers, Academic Press, 2009. 